$\S=-1$

\title{
Power Measurement by Calorimetric Method Using Water Infrequency Range Between 1.7 Mhz To $3 \mathrm{Mhz}$
}

\author{
F. Shokrollahi' ${ }^{1}$ K. K. Lau ${ }^{2 *}$, W. H. Tay ${ }^{3}$, L. S. Lai ${ }^{4}$ \\ ${ }^{1,2,3}$ Department of Chemical Engineering, Universiti Teknologi PETRONAS, Malaysia \\ ${ }^{4}$ Department of Chemical Engineering, University of Illinois at Chicago, US \\ *Corresponding author E-mail: laukokkeong@utp.edu.my
}

\begin{abstract}
Absorption is one of the most established processes for $\mathrm{CO}_{2}$ capturing. However, the current technologies used in this process suffer from several drawbacks. Meanwhile, the ultrasound technique is proposed as a new alternative technology to assist the $\mathrm{CO}_{2}$ absorption process due to both its physical and chemical effects. Yet, the chemical effect is still under developments. Between all the influencing parameters, the ultrasonic power and the frequency are the vital key parameters to investigate sonochemical effects during the $\mathrm{CO}_{2}$ absorption process. The aim of this paper is to measure the ultrasonic power of an ultrasonic vessel. The total electrical power measured by using the voltage and the flow. Simultaneously, the ultrasonic power determined by using the calorimetric method. The measurements were done by using water with different volumes and repeated for three different frequencies. The results showed that almost $51 \%$ of the electrical power converted into the ultrasonic power and were independent of liquid volume.
\end{abstract}

Keywords: Absorption, Electrical power, Sonochemical effect, Ultrasonic power, Ultrasound technology.

\section{Introduction}

Due to the population rise and industrial development global energy demand is increasing. Therefore, it is essential to raise the energy supplies in a way which is affordable, safe and more importantly environmentally- friendly. Nowadays, fossil fuels including natural gas, oil and coal provide almost $85 \%$ of the total energy demand. Due to the availability and abundance, these energy sources play an essential role in the global energy economy. However, they are also known as the main reason for the greenhouse gas emissions, especially $\mathrm{CO}_{2}$ emissions. Meanwhile, total $\mathrm{CO}_{2}$ emission can be reduced through three main options including energy intensity reduction, carbon intensity reduction, and improvement $\mathrm{CO}_{2}$ separation processes. The first option needs efficiently energy use. The second option needs shifting to use non-fossil fuels like renewable or nuclear energy and the third option includes technologies development in order to capture and separate more $\mathrm{CO}_{2}$. This option provides a mid-term solution for decreasing the environmental influences and allows human to continue using fossil energy sources till the nuclear or renewable energy technologies become mature $[1,2]$. Between all the conventional technologies to capture $\mathrm{CO}_{2}$, absorption is the most matured and established one while, the existing contactor systems used in absorption technologies suffer from several drawbacks including excessive footprint (e.g. packed bed column) and operating/maintenance issues (e.g. membrane contactor has pressure and solvent viscosity limitation) [3-6].

Recently, ultrasonic irradiation as a common technique which used in the improvement of multiphase chemical reactions has been suggested as a new alternative technology to help this process mostly due to its physical effect [7].

But, rather than the ultrasonic physical effect, the presence of ultrasonic chemical effect could also improve the chemical reaction of the absorption process. Nevertheless, relevant literature focusing on sonochemical effect during ultrasonic $\mathrm{CO}_{2}$ absorption are scarcely reported. Hence, as the first step for elucidating the sonochemical effect on the $\mathrm{CO}_{2}$ absorption process, ultrasonic power measurement needs to accomplish.

\section{Theoretical Aspects of Ultrasonic Irradia- tion}

Ultrasonic irradiation which is defined as a sound field with the frequency above $20 \mathrm{kHz}$, can be transmitted through any substance having elastic properties. In general, the vibration of the sound source is transferred to the medium molecules and each molecule transmits this motion to the near molecule before turning back to its original position. In liquids, ultrasonic irradiation generates regions with low and high pressure which is known as periodic expansion (rarefaction) and compression. The rarefaction cycle applies the negative pressure which pulls the molecules apart while the compression cycle creates the positive pressure and pushes the liquid molecules together, as shown in Figure. 1. $[8,9]$.

When ultrasonic intensity is sufficiently high, the expansion cycles can create bubbles or cavities inside the liquid which varies depending on the liquid purity and its nature. Since pure liquids have significant tensile strengths, the ultrasonic generators cannot create high enough negative pressures which result in cavitation. While in impure liquids, the tensile strength is decreased because of the presence of solids, dissolved gases, many small particles or any other impurities. These impurities create some weak points inside the liquid medium where nucleation of the cavitation can happen. For instance, in the pure water, above 1000 atm of negative pressure might be needed for the cavitation. While in tap 
water, only a few atmospheres of the negative pressure will form bubbles $[10,11]$.

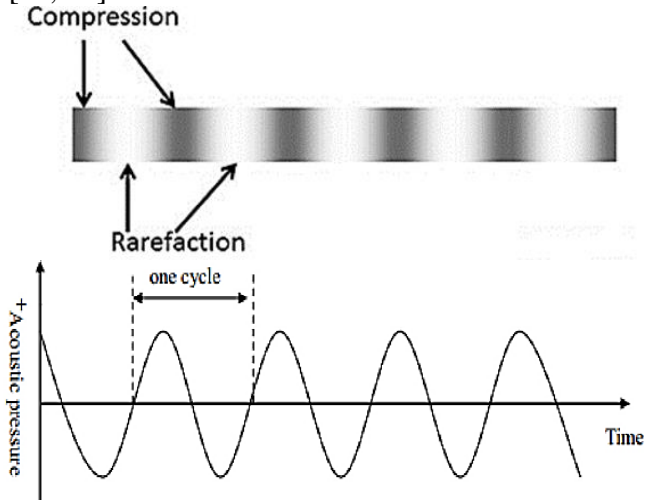

Fig. 1: Ultrasound wave [9]

After the bubble formation, the bubble size might be raised till the maximum of the negative pressure has been reached. Figure. 2 illustrates the formation, growth and implosive collapse of cavitation bubbles.
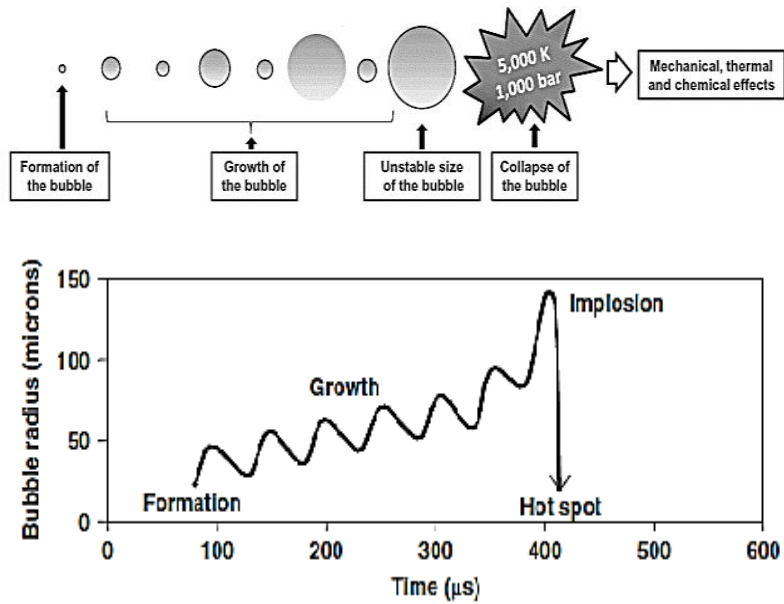

Fig. 2: Bubble formation, growth and collapse [11,12]

Depending on the frequency, there is a critical size for the cavitation bubble in which the cavity absorbs energy from the ultrasound source more efficiently and grows more quickly. But, when a cavity experiences absolutely quick growth it can no longer absorb energy as efficiently from the sound waves and therefore the liquid rushes in and the cavity implodes.

Then, vapors and gas are compressed inside the cavity and this process generates heat which finally produces a short-lived localized hot spot. Finally, the hot spot creates an unusual environment for the chemical reactions in terms of enormous local temperatures and pressures [11-13].

These high pressures and temperatures provide the activation energy needed for the chemical bond breakage. Therefore, highly reactive free radicals and various other species are produced. The produced free radicals can diffuse into the liquid bulk or even react with each other to form new radicals or molecules which can affect the chemical reactions. Accordingly, the most basic concept of sonochemical effect is free radicals form as a result of the cavitation of bubbles and cause the enhancement of the chemical kinetic reaction rate [14-16]. In aqueous solutions between all the radicals the hydroxyl radical $\left(\mathrm{OH}^{\circ}\right)$ is known to initiate a number of reactions which can be studied by measuring changes in the conductivity [17, 18]. Between all the influencing parameters, ultrasonic power and frequency are the key parameters for investigating the sonochemical effects during the $\mathrm{CO}_{2}$ absorption process.

\section{Methodology}

\subsection{Apparatus}

As shown in Figure. 3, the experiments are conducted in a stainless steel batch vessel. The vessel has two main parts including a top cover and cylindrical body. The diameter, height and the total volume of the vessel are $5 \mathrm{~cm}, 17.8 \mathrm{~cm}$ and $350 \mathrm{ml}$, respectively. The top cover with five outlet ports used to mount one temperature and one pressure sensor, one inlet and outlet gas pipeline as well as one conductivity probe. Since the formation of $\mathrm{OH}^{*}$ radicals would affect the conductivity, in-situ conductivity measurement can be a possible option to clarify the role of radical generated during the $\mathrm{CO}_{2}$ absorption process.

For direct contact with the solvent, the ultrasonic transducer is located at the bottom of the vessel and converts the electrical power to ultrasonic irradiation. Three different transducers with the same diameter of 1.5 but different frequencies, $1.7 \mathrm{MHz}$, $2.4 \mathrm{MHz}$ and $3 \mathrm{MHz}$ have been used.

The vessel will be filled by $100 \mathrm{ml}$ of different solvents with different concentrations. During the experiments, the temperature of the solvent might increase slightly because of the power dissipated by ultrasonic irradiation. Therefore, the vessel should settle in a water bath in order to control the temperature variation of the solvent. Next, pure $\mathrm{CO}_{2}$ gas is compressed to the vessel. At the same time, the mass flow controllers can control the gas mixture composition. After that, adjusting the position of valve 1 and 2 along with a back pressure regulator enable us to pressurize and control the pressure of the ultrasonic vessel. Finally, the experimental procedures can be run for different solvents and operation parameters

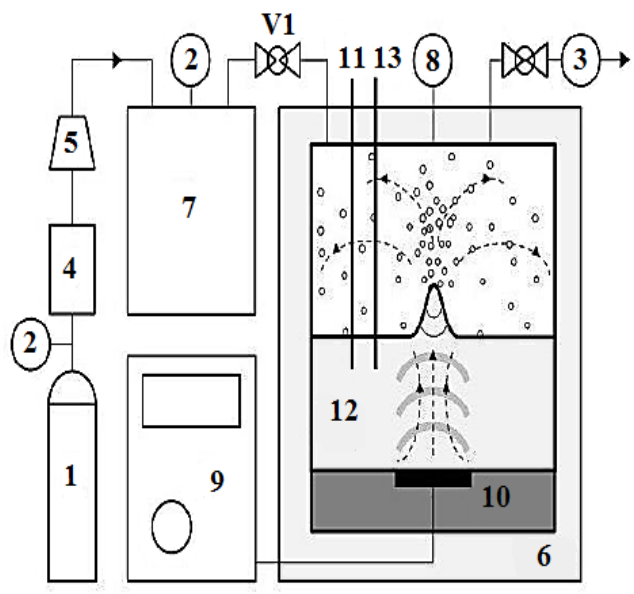

\begin{tabular}{|c|l|c|l|}
\hline 1 & $\mathrm{CO}_{2}$ gas tank & 8 & Pressure sensor \\
\hline 2 & Pressure gauge & 9 & Ultrasonic power system \\
\hline 3 & Back pressure regulator & 10 & Ultrasonic transducer \\
\hline 4 & Mass flow controller & 11 & Temperature sensor \\
\hline 5 & Compressor & 12 & Ultrasonic vessel \\
\hline 6 & Water bath & 13 & Conductivity probe \\
\hline 7 & Gas storage vessel & V1, & Valve 1, Valve 2 \\
\hline
\end{tabular}

Fig. 3: Schematic diagram of experiment setup

Since the performance of the ultrasonic-assisted absorption system is strongly depended on the ultrasonic power, it needs to be elucidated and measured accurately. The principals of the ultrasonic power measurement are described in the following section. 


\subsection{Transmitted Power Measurement}

By using ultrasonic irradiation as an external energy source to trigger the reactions, it is essential to calculate the power transmitted by it to the medium. Besides, the ultrasonic power is the output of the ultrasonic system while the total power is the input of the electrical power system. The total electrical power can be directly measured by using Eq. 1 .

\section{$\mathrm{P}_{\mathrm{T}}=\mathrm{VI}$}

Where, $\mathrm{P}_{\mathrm{T}}$ is total electrical power, $\mathrm{V}$ is the $\mathrm{DC}$ voltage which supplied to the circuit, and I is the DC current flow.

Ultrasonic power can be measured via several methods such as calorimetric, radiation force and optical diffraction. Among these three methods, probably the first method is the most common due to the accuracy and simplicity of operation [19-21]. According to the calorimetric method, ultrasonic power $\left(\mathrm{P}_{\mathrm{US}}\right)$ can be determined by measuring the rate of temperature rise because of the changing the ultrasound energy into heat and calculating by using Eq. 2 .

$$
\mathrm{P}_{\mathrm{US}}=\mathrm{V}_{\mathrm{l}} \rho_{\mathrm{l}} \mathrm{C}_{\mathrm{pl}} \mathrm{dT} / \mathrm{dt}
$$

In Eq. 2, $V_{1}$ is the volume of the liquid, $\rho_{1}$ is the liquid density, $C_{p l}$ is the liquid heat capacity and $\mathrm{dT} / \mathrm{dt}$ is temperature increasing rate which can be recorded by using data acquisition system. Figure. 4 shows the schematic diagram of the calorimetric method.

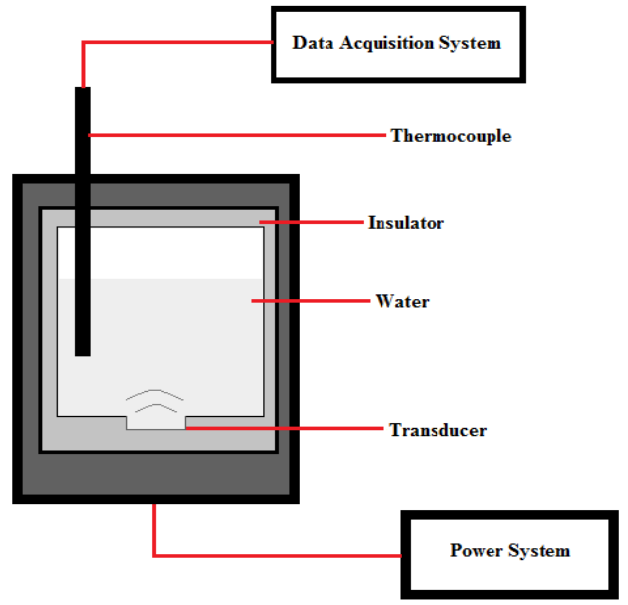

Fig. 4: Power measurements schematic diagram using calorimetric method

The correlation between the ultrasonic power and the total electrical power can be expressed by using Eq. 3 .

$\mathrm{P}_{\mathrm{US}}=\mathrm{kP}_{\mathrm{T}}$

Where, $\mathrm{k}$ is the coefficient of the ultrasonic power to the total electrical power.

In this study during a specific time, the power measurement has been done by using water as a solvent with two different volume. The experiments have been reapeted at three different frequencies including $1.7 \mathrm{MHz}, 2.4 \mathrm{MHz}$, and $3 \mathrm{MHz}$, respectively.

\section{Results \& Discussion}

As mentioned earlier, the temperature increasing rate must be determined as the first step in clarifying the correlation between the total electrical power and the ultrasonic power. Figure. 5 shows how the temperature increases under the ultrasonic irradiation at various ultrasonic applied voltages by using $100 \mathrm{ml}$ distilled water which is irradiated at three different frequencies. It is worth to note that the temperature value has been recorded using data acquisition system.

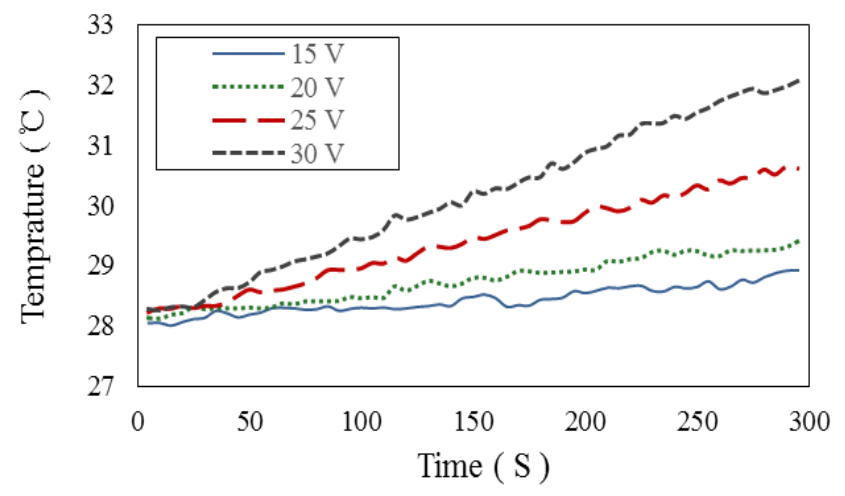

(a)

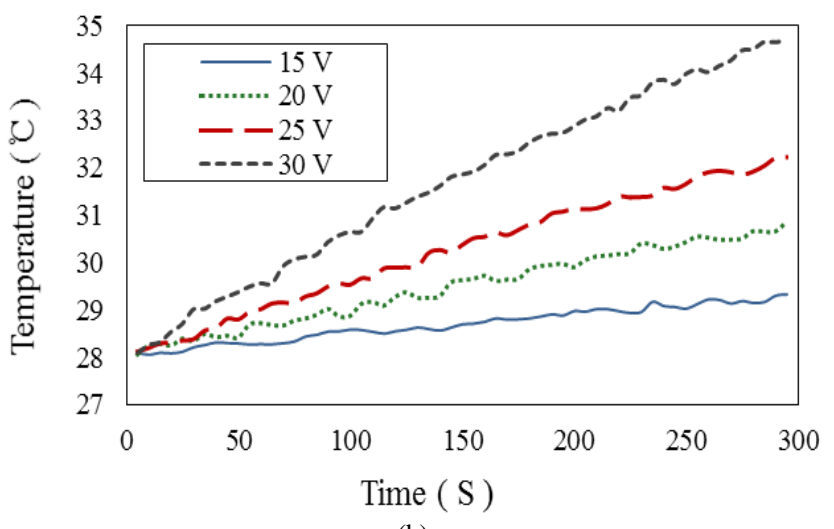

(b)

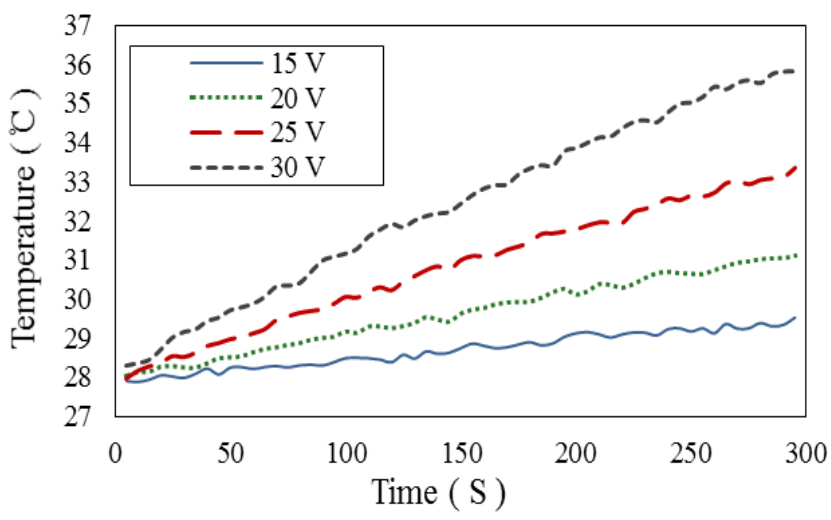

(c)

Fig. 5: Temperature profiles at different applied voltage (a) $1.7 \mathrm{MHz}$, (b) $2.4 \mathrm{MHz}$, (C) $3 \mathrm{MHz}$

According to the results, it can be seen that by increasing the applied voltage the temperature is also increasing. Besides, the increase rate in temperature amount is faster when higher frequencies are applied. The liquid temperature rise which is more significant at higher frequencies can reduce the cavitation thresholds as a result of the increase in the liquid vapor pressure or a decrease in surface tension or even viscosity. Therefore, by applying higher frequencies the number, size, and distribution of the cavitation bubbles can be affected. In the other word, higher frequencies produce a greater number of cavitation bubbles that can collapse violently and result in an increase in the temperature. Moreover, it can be expected that at higher frequencies there would be a greater propensity for chemical reactions. As frequency rises, the amount of vapour entering the bubble decreases due to the reduction in its expansion phase. The increased rate of oscillation due to using higher frequencies and reduction in bubble growth per cycle allows small bubbles to reach critical size more efficiently than lower frequencies and then implodes. In addition, as noted previously, each implosion can create an unusual environment for a chemical reaction in terms of 
enormous local temperatures and pressures. After measuring the temperature increasing rate the desired aim is to find out that how much ultrasonic power is being provided. In this study, the ultrasonic power absorbed by $100 \mathrm{ml}$ of distilled water has been measured calorimetrically at three different applied frequencies. Figure. 6 displays the correlation between the total electrical power and the ultrasonic power. Based on the results it can be seen that higher frequencies can lead to higher ultrasonic power due to the higher temperature increasing rate. Moreover, as noted previously higher ultrasonic power can trigger the bubble formation and cavitation which can influence the chemical effects. Therefore, the most attention would be given to the highest frequency which is $3 \mathrm{MHz}$.

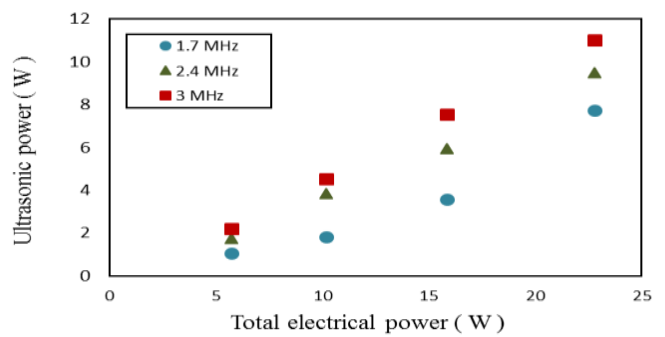

Fig. 6: The correlation between total electrical power and ultrasonic power at different applied frequencies

After selecting properly the most appropriate frequency which is 3 $\mathrm{MHz}$, it is important to find out the influences of the liquid amount on the power transmission. For this aim, two different volume of the water as the cavitating medium were examined. Figure. 7 displays the electrical power and ultrasonic power correlation at $3 \mathrm{MHz}$.

According to the results, it can be figured out that higher power output of the electrical power system produces higher ultrasonic power. Moreover, the ultrasonic power coefficient, $\mathrm{k}$, is equal to $0.51 \pm 0.01$. It means that approximately $51 \%$ of the electrical power has been converted into the ultrasonic power.

Based on the transducer specifications, this power transmission is accepted. Also, the transmission was found to be independent on the amount of liquid. It is believed that most of the ultrasonic power has been absorbed in the liquid through the ultrasonic power absorption and the vibration of the gas-liquid interfacial layer.

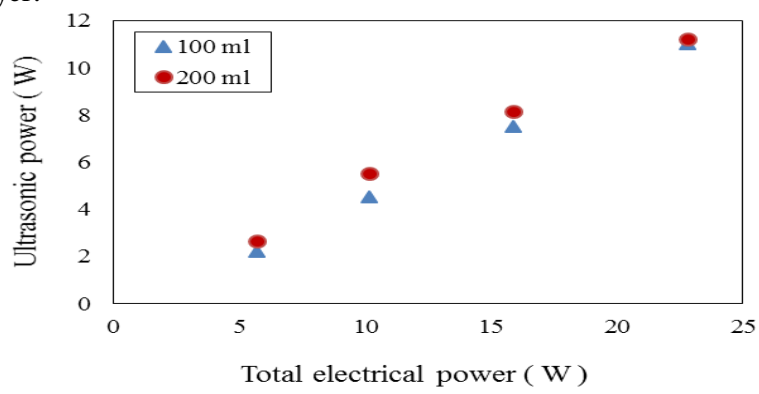

Fig. 7: Electrical power and ultrasonic power correlation at $3 \mathrm{MHz}$

\section{Conclusion}

Since the performance of the ultrasonic-assisted $\mathrm{CO}_{2}$ absorption system depends on the ultrasonic power, it needs to be elucidated and measured accurately. In the current work after choosing the best frequency due to the higher temperature increase rate, the ultrasonic power measured using the calorimetric method. The results showed that almost $51 \%$ of electrical power converted into ultrasonic power which is an acceptable amount based on the transducer specifications. Moreover, under the experimental conditions, it was found that power transmission is almost independent of the liquid volume.

\section{Acknowledgement}

The author greatly appreciates the support provided by University Teknologi PETRONAS for this research.

\section{References}

[1] S. Kumar, J. H. Cho, and I. Moon, "Ionic liquid-amine blends and $\mathrm{CO}_{2}$ BOLs: prospective solvents for natural gas sweetening and $\mathrm{CO}_{2}$ capture technology-a review," International Journal of Greenhouse Gas Control, vol. 20, pp. 87-116, 2014.

[2] H. Yang et al., "Progress in carbon dioxide separation and capture: A review," Journal of environmental sciences, vol. 20, no. 1 , pp. 14-27, 2008.

[3] F. Chu, C. Jon, L. Yang, X. Du, and Y. Yang, "CO $\mathrm{CO}_{2}$ absorption characteristics in ammonia solution inside the structured packed column," Industrial \& Engineering Chemistry Research, vol. 55, no. 12, pp. 3696-3709, 2016.

[4] L. Tan, A. Shariff, K. Lau, and M. Bustam, "Impact of high pressure on high concentration carbon dioxide capture from natural gas by monoethanolamine/N-methyl-2-pyrrolidone solvent in absorption packed column," International Journal of Greenhouse Gas Control, vol. 34, pp. 25-30, 2015

[5] C. Makhloufi, E. Lasseuguette, J. C. Remigy, B. Belaissaoui, D. Roizard, and E. Favre, "Ammonia based $\mathrm{CO}_{2}$ capture process using hollow fiber membrane contactors," Journal of membrane science, vol. 455, pp. 236-246, 2014.

[6] Z. Ze and J. Sx, "Hollow fiber membrane contactor absorption of $\mathrm{CO}_{2}$ from the flue gas: review and perspective," Glob. Nest J., vol. 16 , pp. 355-374, 2014.

[7] W. Tay, K. Lau, and A. Shariff, "High frequency ultrasonic-assisted chemical absorption of $\mathrm{CO}_{2}$ using monoethanolamine (MEA)," Separation and Purification Technology, vol. 183, pp. 136-144, 2017.

[8] X. Cheng, M. Zhang, B. Xu, B. Adhikari, and J. Sun, "The principles of ultrasound and its application in freezing related processes of food materials: A review," Ultrasonics sonochemistry, vol. 27, pp. 576-585, 2015.

[9] K. Yasui, Acoustic Cavitation and Bubble Dynamics. Springer, 2018.

[10] T. Y. Wu, N. Guo, C. Y. Teh, and J. X. W. Hay, Advances in ultrasound technology for environmental remediation. Springer Science \& Business Media, 2012.

[11] F. P. Capote and M. L. de Castro, Analytical applications of ultrasound. Elsevier, 2007.

[12] P. Chowdhury and T. Viraraghavan, "Sonochemical degradation of chlorinated organic compounds, phenolic compounds and organic dyes-a review," Science of the total environment, vol. 407, no. 8 , pp. 2474-2492, 2009.

[13] J. E. Crooks and J. P. Donnellan, "Kinetics and mechanism of the reaction between carbon dioxide and amines in aqueous solution," Journal of the Chemical Society, Perkin Transactions 2, no. 4, pp. 331-333, 1989.

[14] Y. G. Adewuyi, "Sonochemistry: environmental science and engineering applications," Industrial \& Engineering Chemistry Research, vol. 40, no. 22, pp. 4681-4715, 2001.

[15] P. Riesz and T. Kondo, "Free radical formation induced by ultrasound and its biological implications," Free Radical Biology and Medicine, vol. 13, no. 3, pp. 247-270, 1992.

[16] L. Thompson and L. Doraiswamy, "Sonochemistry: science and engineering," Industrial \& Engineering Chemistry Research, vol. 38, no. 4, pp. 1215-1249, 1999.

[17] A. Diplock, M. C. Symons, and C. Rice-Evans, Techniques in free radical research. Elsevier, 1991.

[18] A. R. Jambrak, T. J. Mason, V. Lelas, Z. Herceg, and I. L. Herceg, "Effect of ultrasound treatment on solubility and foaming properties of whey protein suspensions," Journal of Food Engineering, vol. 86, no. 2 , pp. 281-287, 2008

[19] T. Delchar and R. Melvin, "A calorimeter for ultrasound total power measurements," Measurement Science and Technology, vol. 5, no. 12 , p. 1533,1994

[20] T. Uchida, T. Kikuchi, M. Yoshioka, Y. Matsuda, and R. Horiuchi, "Development of high ultrasonic power measurement technique by calorimetric method using water as heating elements for high ultrasonic power standard," Acoustical Science and Technology, vol. 36 , no. 5 , pp. 445-448, 2015.

[21] T. Uchida and T. Kikuchi, "1P2-3," in Proceedings of Symposium on Ultrasonic Electronics, 2013, vol. 34, no. 2013, pp. 41-42. 